\title{
Research on a detection algorithm for abnormal state of load
}

\author{
Dan $\mathrm{Yu}^{1,2^{*}}$, Yubo Wang ${ }^{1,2}$, Jun $\mathrm{Du}^{1,2}$, Lizong Liu ${ }^{1,2}$, Haojie Tian $^{3}$, Yan Guan ${ }^{3}$ and Shengfang Li ${ }^{1,2}$ \\ ${ }^{1}$ State Grid Key Laboratory of Power Industrial Chip Design and Analysis Technology, Beijing Smart-Chip Microelectronics \\ Technology Co., Ltd. Beijing 100192, China \\ ${ }^{2}$ Beijing Engineering Research Center of High-reliability IC with Power Industrial Grade, Beijing Smart-Chip Microelectronics \\ Technology Co., Ltd. Beijing 100192, China \\ ${ }^{3}$ State Grid Liaoning Electric Power Supply Co., Ltd. Liaoning 110006, China
}

\begin{abstract}
This paper describes the development status and main problems of load monitoring, introduces the key technologies of load monitoring, and by using a load state monitoring system, emphatically illustrates a detection algorithm for abnormal state of the secondary load of the current transformer on the three-phase line of the power grid. The algorithm mainly achieves the real-time detection of abnormal state such as disconnection, short connection and series connected semiconductor. In the light of this algorithm, the working principle is explained, the model formula is worked out, and the state criterion is given. The load condition monitoring system is debugged, put into operation and tested in the pilot operation, and the results show that the algorithm has a good effect.
\end{abstract}

\section{Introduction}

With the development of national economy, electric energy has become an indispensable energy source in daily production and life. However, abnormal state of the power load in our country is common and serious, which brings huge economic losses.

The combination of key technical measures such as network technology, sensor technology, communication technology, and information technology makes load monitoring of our country change from conventional passive way to all-weather, all-round, real-time active and intelligence way.

At present, load monitoring is divided into two types of intrusive and non-intrusive [1-2]. In the past, intrusive load monitoring was used, that is, sensors and other information acquisition devices which are installed at the load to obtain load information. As the market of load monitoring is increasingly expanding, non-intrusive load monitoring (NILM [3]) is proposed and widely concerned which is compared with the traditional intrusive [4-5]. The installation of information acquisition devices is at the entrance of the monitored power area to obtain load information, such as current and power. Based on these, the situation of each load in the area is analyzed. Compared with intrusive load monitoring, non-intrusive load monitoring has the advantages of simple installation, good economy, high reliability and easy to popularize $[1,6]$, and is suitable for independent user installation mode $[1,7]$.

In order to discover and deal with abnormal state of the secondary load of the current transformer on the three-phase line of the power grid (usually including disconnection, short connection and series connected semiconductor), the load state monitoring system is developed to make up for the lack of existing measures. The load state monitoring system ensures the normal operation of the power grid, so that non-intrusive load monitoring is achieved smoothly.

According to the requirement of load state monitoring system, a detection algorithm based on analysis of self oscillation and $\mathrm{AC}$ current harmonics is studied to identify the abnormal state of secondary load by using FFT signal processing technology and impedance model of the secondary load. The algorithm is small in complexity, the identification state is accurate and the validity is verified by practice, so that the load state monitoring system achieves real-time, accurate, intuitive and comprehensive monitoring of the secondary load state.

\section{Description of the detection algorithm}

\subsection{Detection principle of disconnection, short connection}

A resonant circuit is constructed by using isolated magnetic couplers and capacitors, which is incorporated into the secondary load circuit for forming self-excited oscillation.

Self-excited oscillation is the basis for detecting abnormal disconnection and short connection. These abnormal states of the secondary load will show the abrupt change of the secondary load impedance, and this change will be directly expressed as the change of selfexcited oscillation frequency. This self-excited

\section{Corresponding author: ewebbac@163.com}


oscillation frequency becomes high in short circuit and it becomes low in open circuit.

If the measurement accuracy of this frequency is strictly required, the detection of the abnormal disconnection will become a problem. Therefore, it is necessary to inject an $\mathrm{AC}$ signal with the appropriate frequency band to assist identification, and the corresponding signal pickup circuit is also needed.

\subsection{Analysis of temperature compensation during open circuit and short circuit}

In the practical application, it is found that the isolated magnetic coupler for self-excited oscillations has a temperature characteristic which affects the self-excited oscillation frequency, because the permeability of isolated magnetic coupler core material will change greatly when the ambient temperature changes.

In this case, the temperature experiment of the highprecision liquid bath was completed for the isolated magnetic coupler composed of MnZn ferrite and ultramicrocrystalline (nanocrystalline). In this experiment, the temperature was kept stable for half an hour at different temperatures, and changes in the parameters was measured by the standard LCR tester. LCR tester measurement model: Ls-Q (series equivalent), the test level is $1 \mathrm{~V}$.

The parameter of the corresponding magnetic core inductance is leakage inductance in short circuit, and it is self inductance in open circuit.

When abnormal disconnection or short connection is happened in the secondary load circuit, the temperature characteristics of ferrite material parameters are shown in Figure 1 or Figure 2. The curves of 5, 15, 20 and 30 in Figure 1 and Figure 2 show the values measured at the test frequencies of the LCR tester $(5 \mathrm{kHz}, 15 \mathrm{kHz}, 20 \mathrm{kHz}$, and $30 \mathrm{kHz}$, respectively).

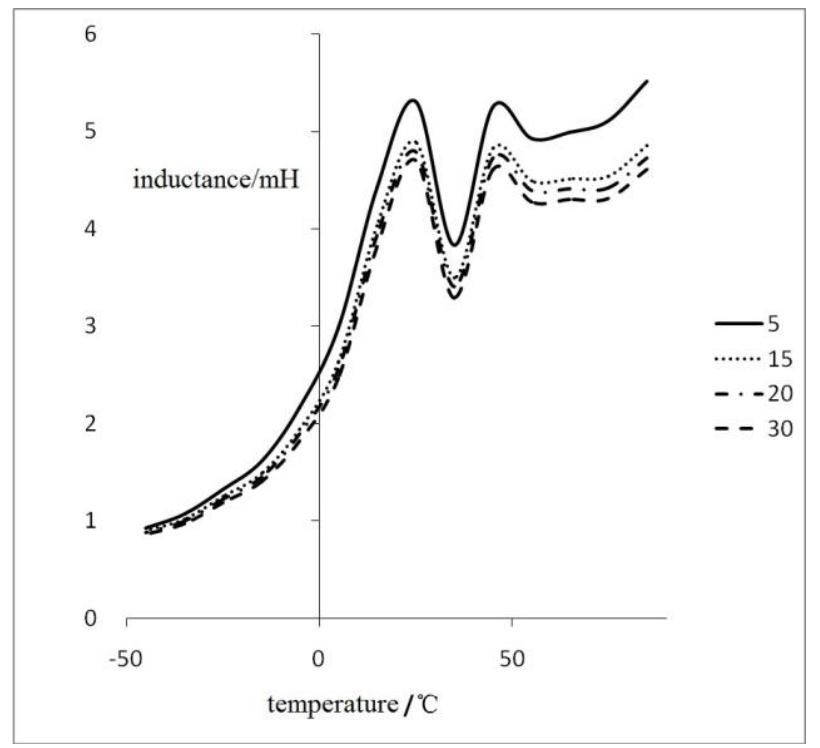

Fig. 1. The self inductive parameters of ferrite vary with temperature.

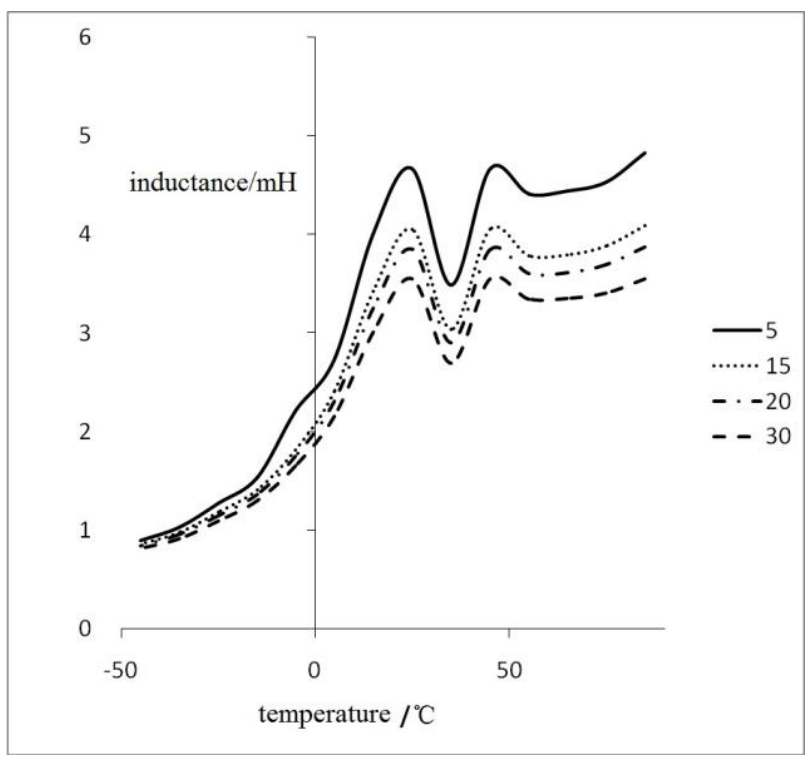

Fig. 2. The leakage inductive parameters of ferrite vary with temperature.

The characteristics of ferrite material parameters are shown in Figure 1 and Figure 2:

- Large leakage inductance (self inductance and leakage inductance are not much difference);

- Occurrence of the diving fluctuation, $25^{\circ} \mathrm{C} \sim 45^{\circ} \mathrm{C}$;

- Basic stability (there is a certain upward trend), above $50^{\circ} \mathrm{C}$;

- The downward trend of the approximate quadratic function curve, below $25{ }^{\circ} \mathrm{C}$.

When abnormal disconnection or short connection is happened in the secondary load circuit, the temperature characteristics of ultra-microcrystalline material parameters are shown in Figure 3 or Figure 4. The curves of 5, 10, 15 20, 25 and 30 in Figure 3 and Figure 4 show the values measured at the test frequencies of the LCR tester $(5 \mathrm{kHz}, 10 \mathrm{kHz}, 15 \mathrm{kHz}, 20 \mathrm{kHz}, 25 \mathrm{kHz}$ and $30 \mathrm{kHz}$, respectively).

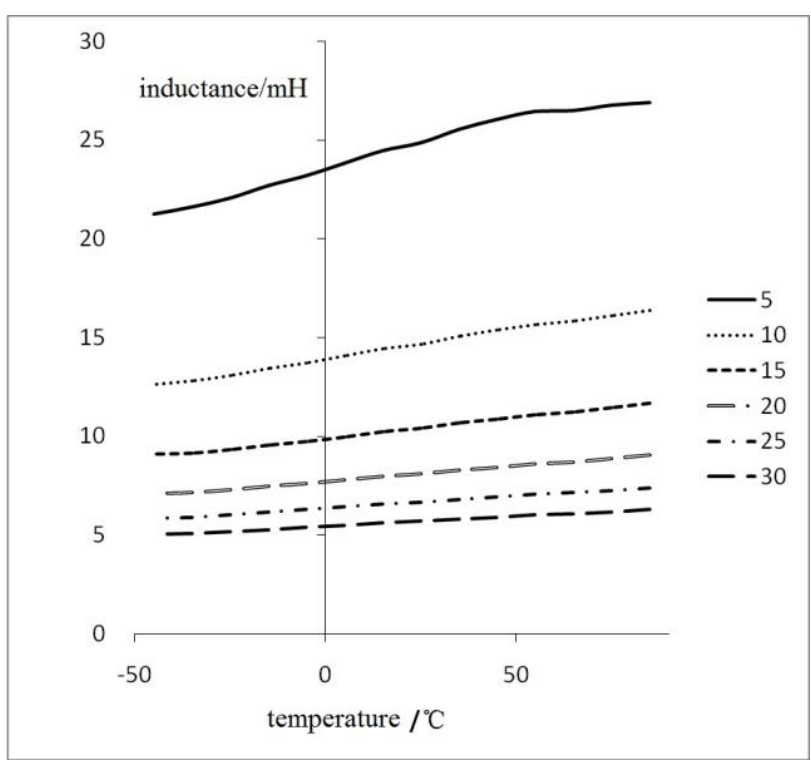

Fig. 3. The self inductive parameters of ultra-microcrystalline vary with temperature. 


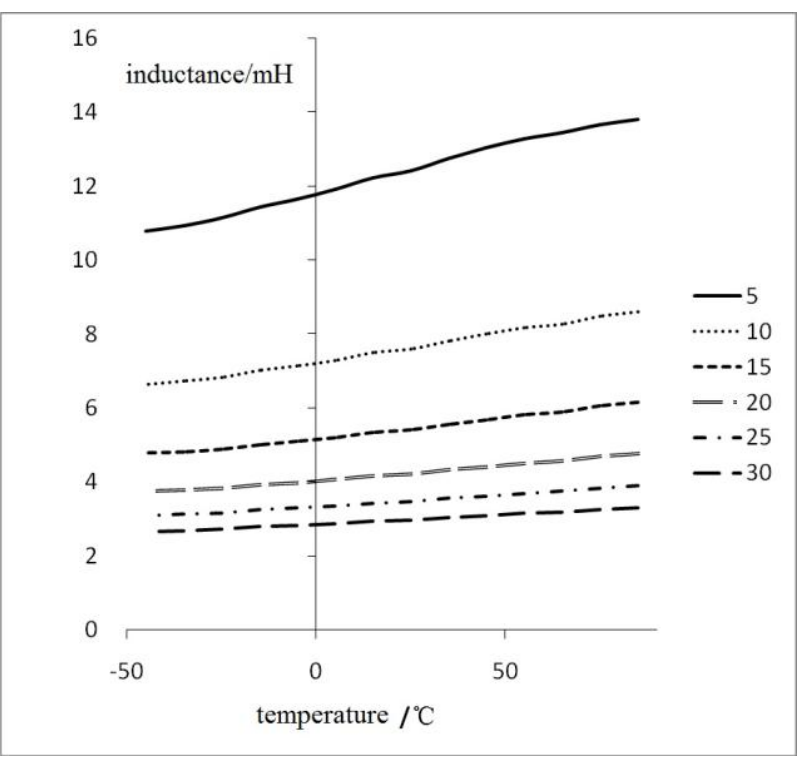

Fig. 4. The leakage inductive parameters of ultramicrocrystalline vary with temperature.

The characteristics of ultra-microcrystalline material parameters are shown in Figure 3 and Figure 4:

- high permeability, small leakage inductance;

- almost linear variation with temperature;

- the slope with the change of temperature is small (relatively gently).

Thus, different temperature compensation methods should be given for different coupling element material characteristics, and for ferrite should be segmented compensation, whereas for nanocrystalline only linear compensation is needed.

The temperature compensation is realized by the curve fitting method based on the least square principle, The regression analysis model is constructed by linear equation and polynomial.

\subsection{Detection principle of series connected semiconductor}

The semiconductor described in this paper refers to the diode. Due to the unidirectional conductivity of the diode, the load current changes from the pure AC signal to the pulsating DC signal after the semiconductor is connected in the secondary load circuit.

The key to the detection algorithm of series connected semiconductor is how to calculate and analyze the waveform characteristics of the secondary load current in the abnormal state by using sampled series current data.

Fourier spectrum analysis is the key to the analysis of the load current waveform in the abnormal state of series connected semiconductor [8].

According to the Fourier transform [9]:

$$
\stackrel{\Lambda}{f(\xi)}=\int_{0}^{\frac{1}{\xi}} f(t)^{-i 2 \pi \xi t} d t
$$

In formula (1): $f(t)$ - time domain function; $f(\xi)$ frequency domain function.

In the generalized the Fourier transform, the upper and lower bounds of the integral cycle should be $-\infty \sim+$ $\infty$, but only $1 / \xi$ is used, because it is no necessary to consider the effects of subharmonics and non-integer harmonics, and the calculation is convenient. $1 / \xi$ is the minimum integral cycle, of course, you can also take an integer multiple of $1 / \xi$.

Further solving vector $(\hat{f(\xi)})$ : according to Euler's formula, put $e^{-i 2 \pi \xi t}=\cos 2 \pi \xi t-i \sin 2 \pi \xi t$ into formula (1), then formula (2) is formed.

In order to express the relationship between science between quantity and degree, and convenient program portability, engineering calculation will usually be normalized to the number of numerical and harmonic content into a normalized value, then processing.

$$
\begin{aligned}
& \stackrel{\Lambda}{f(\xi)}=\int_{0}^{\frac{1}{\xi}} f(t) \cos 2 \pi \xi t d t-i \int_{0}^{\frac{1}{\xi}} f(t) \sin 2 \pi \xi t d t \\
& \text { In formula (2): } \int_{0}^{1 / \xi} f(t) \cos 2 \pi \xi t d t \quad-\text { cosine }
\end{aligned}
$$

component of harmonic frequency; $\int_{0}^{1 / \xi} f(t) \sin 2 \pi \xi t d t-$ sinusoidal component of harmonic frequency.

In order to scientifically demonstrate the relationship between quantity and degree, and facilitate the transplant of the program, many numerical values are usually normalized in engineering calculation, that is, the above harmonic content is converted to the per-unit value, and then processed.

Due to the similar "fingerprint" feature of such harmonics is fixed at the ratio of the even harmonic spectral density (except the fundamental wave), the ratio of the adjacent even harmonic spectral density can be used to confirm "Fingerprint" features of current harmonics in the abnormal state of series connected semiconductor. Since the higher harmonic content is usually very small, and the cost of the system sampling rate and calculation is high, the lower harmonics are selected to be solved. Because of the limitation of space, the solution process is omitted. The following are three criteria for series connected semiconductor:

$$
\begin{aligned}
& R_{21}=\frac{\left|U_{2 b}\right|}{\left|U_{b}\right|}=42.44 \% \\
& R_{42}=\frac{\left|U_{4 b}\right|}{\left|U_{2 b}\right|}=20.03 \% \\
& R_{64}=\frac{\left|U_{6 b}\right|}{\left|U_{4 b}\right|}=42.82 \%
\end{aligned}
$$


In formula (3), (4), (5): $U_{b}$ - Fundamental value; $U_{2 b}$ - Second harmonic value; $U_{4 b}$ - Fourth harmonic value; $U_{6 b}$ - Sixth harmonic value.

If the formula (1), (2), (3) are true, the abnormal state of series connected semiconductor is detected.

If the formula (2), (3) are true, and the equation (1) is false, it is detected that there is the half-wave direct current component in the primary current of the current transformer on the three-phase line of the power grid.

Note: In formula (1), $42.44 \%$ is the maximum value. When there is a part of the DC component, the value of this item will be small or even close to zero.

\subsection{Saturation analysis of magnetic circuit in series connected semiconductor}

In the practical application, it is found that when the secondary load circuit is greater than a certain value, the core of the current transformer on the three-phase line of the power grid enters the saturation state; when the secondary load circuit is greater than a larger value, then it enters the deep saturation state.

After the semiconductor is connected in the secondary load circuit, the effect of magnetic saturation caused by the DC bias is shown in Figure 5. At this point, parameters of the current transformer on the three-phase line of the power grid: the change ratio is 150: 5; the maximum secondary load is $5 \mathrm{VA}$; the accuracy level is $0.2 \mathrm{~S}$.

The measured current in Figure 5 is the measured value obtained by CPU processing after sampling the secondary load current with an analog-to-digital converter. The input current is the actual value of the secondary load current.

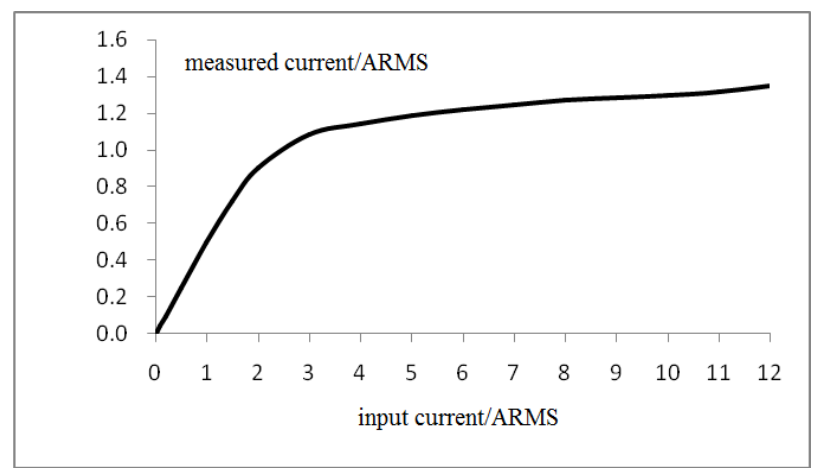

Fig. 5. The relationship between measured current and input current.

After the semiconductor is connected in the secondary load circuit, the current waveform varies with the secondary load current as shown in Figure 6, Figure 7, Figure 8, and Figure 9.

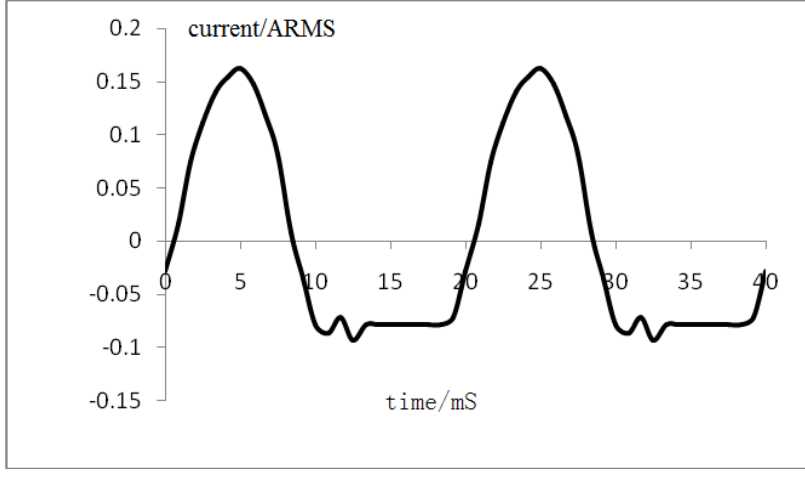

Fig. 6. In the half wave excitation, the waveform of the input current varies. (The input current is $200 \mathrm{~mA}$ )

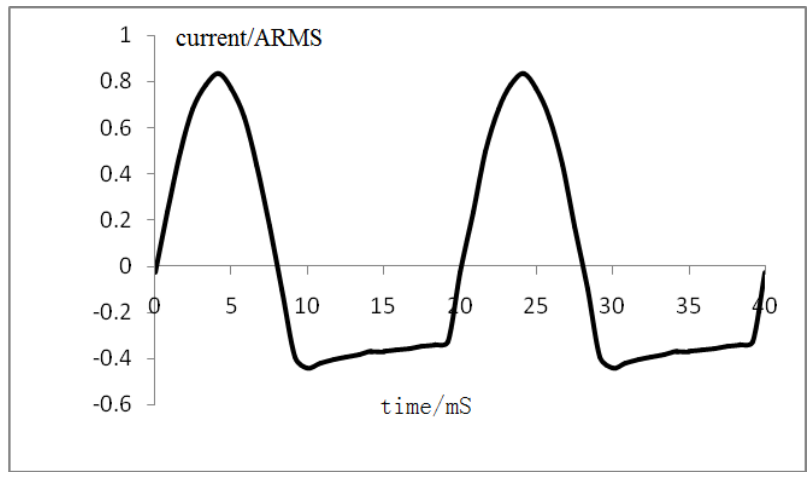

Fig. 7. In the half wave excitation, the waveform of the input current varies. (The input current is $1 \mathrm{~A}$ )

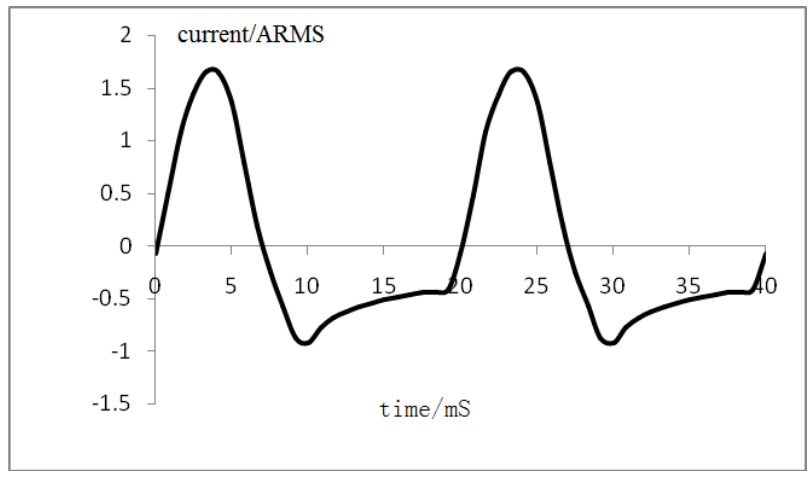

Fig. 8. In the half wave excitation, the waveform of the input current varies. (The input current is $2 \mathrm{~A}$ )

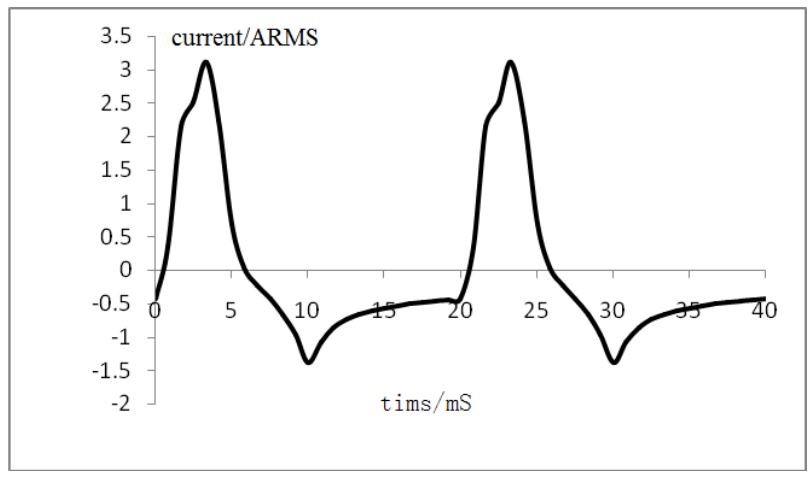

Fig. 9. In the half wave excitation, the waveform of the input current varies. (The input current is $4 \mathrm{~A}$ )

Analysis and comparison of the waveform changes of the secondary load current: 
- When the current is less than 2A, the current waveform basically conforms to the ideal half wave model. Among them, the half wave in Figure 6 should be the closest ideal (the bottom of the half-wave is flat), the bottom of the half wave in Figure 7 appears linearly tilted, and the bottom of the half wave in Figure 8 appears nonlinear tilt.

- When the current is greater than $2 \mathrm{~A}$, the distortion of the current waveform is exacerbated, as shown in Figure 9.

- Because the saturation of magnetic circuit leads to the nonlinear transmission of signals, the current waveform is no longer the superposition of a DC component and even harmonic components, in which odd harmonic components are added.

In order to balance the function and cost, the core saturation of the current transformer on the three-phase line of the power grid is difficult to avoid. In this way, the criterion for detecting the abnormal state of series connected semiconductor can not simply depend on the ratio of adjacent even harmonics. It needs to be combined with the distortion of a certain number of harmonic components.

\section{Conclusion}

This paper studies a detection algorithm for abnormal state of the secondary load, as the core part of the load condition monitoring system to ensure the normal operation of the intrusive load monitoring.

The advantages of the algorithm are as follows:

- The algorithm provides a more practical criterion, and the corrective measures are given in combination with the effects of ambient temperature and magnetic saturation.

- The algorithm has low computational complexity and short calculation time, so it can meet the requirement of real-time.

In addition, the algorithm is simple, effective, stable and easy to maintain.

\section{References}

1. Yu Yixin, Liu Bo, Luan Wenpeng. Nonintrusive Residential Load Monitoring and Decomposition Technology. Southern power system technology, 7, 4 (2013)

2. Liu Bo, Luan Wenpeng. Conceptual Cloud Solution Architecture and Application Scenarios of Power Consumption Data Based on Load Disaggregation. Power System Technology, 40, 3 (2016)

3. Hart G W. Nonintrusive appliance load monitoring. Proceedings of the IEEE, 80, 12 (1992)

4. Drenker S, Kader A. Nonintrusive monitoring of electric loads. IEEE Computer Applications in Power, 12, 4 (1999)

5. Li Peng. Non-intrusive Method for Power Load Disaggregation and Monitoring. Tianjin University Ph.D. Thesis (2009)
6. Gao Yun, Yang Honggeng. Household Load Measurement by Classification Based on Multiscale Wavelet Decomposition. Proceedings of the CSU-EPSA, 27, 7 (2015)

7. Niu Lulu, Jia Hongjie. Transient Event Detection Algorithm for Non-intrusive Load Monitoring. Automation of Electric Power System, 35, 9 (2011)

8. GB/T 17215.321-2008 Electricity metering equipment (a.c.) - Particular requirements - Part 21: Static meters for active energy (class 1 and 2). (IEC 62053-21:2003, IDT)

9. E.Chu, A.George. Inside the FFT Black Box: Serial and Parallel Fast Fourier Transform Algorithms. Canada: CRC Press LLC (2000) 